\title{
La historia como revelación de Dios según Pannenberg. Reflexión crítica.
}

\section{Antonio González F. \\ Centro de Reflexión Teológica, San Salvador.}

Pocos teólogos han pensado con tanta consecuencia y radicalidad como Pannenberg' el significado de la historia para la autocomprensión misma del cristianismo. Según Pannenberg, "la historia es el horizonte abarcador de la teología cristiana. Todas las preguntas y respuestas teológicas tienen sentido solamente dentro del marco de la historia" (HG 218).

Este trabajo tiene por objetivo mostrar en qué sentido concreto la historia es el horizonte aburcador o, por así decirlo, el punto de partida de la teología fundamental de Pannenberg, para en un segundo momento pasar a hacer algunas consideraciones crílicas sobre este punto de partida.

\section{El concepto de teología}

Para abordar la pregunta que aquí nos hemos planteado es menester comenzar por preguntarse por el concepto y la función que este teologo le adscribe a la reflexión teológica.

Para Pannenberg, el tema de la teología no es otro que la verdad de la religión cristiana (cfr. ST 11ss, 58ss), hoy especialmente puesta en tela de jucio por el ateísmo. Esto significa por de pronto la afirmación del carácter fundamentalmente teórico de la teología frente a quienes piensan que ésta es una disciplina práctica por tener por objeco la salvación de los hombres. Pensar asi, dice Pannenberg, llevaria a excluir de la teología el problema de la verdadera realidad de lo divino y a tomar prestada la idea de Dios de otras religiones o filosofías (cfr. ST 13-14) ${ }^{2}$. Solamente si la doctrina cristiana es la verdadera, es posible que ésta tenga algo que decir sobre la salvación de los hombres, pues en definitiva es la convicción de la verdad de la religión cristiana lo que funda la pervivencia de ias iglesias y lo que puede scrvir para justificar y fundamentar 
una determinada praxis de los cristianos (cfr. ST 17).

Pero, ¿qué se entiende concretamente por verdad? Para Pannenberg, la primera característica de la verdad es su falta de contradicciones consigo misma y con todo lo que se considera como verdadero (cfr. ST 29). Esta concepción de la verdad tiene en principio dos consecuencias que serán, como veremos, de enorme trascendencia para la teología de Pannenberg. En primer lugar, verdad significa ante todo coherencia, de modo tal que la consistencia teórica del cristianismo signfica, ya antes de su necesaria confirmación práctica, una primera verificación teórica (cfr. ST 33). Tanto es así, que la correspondencia de un determinado juicio con la realidad juzgada no es para Pannenberg más que una forma especial de la coherencia intema de la verdad, la cual a su vez naturalmente "se vuelve" coherencia de las cosas reales mismas (cfr. ST 63).

Por otra parte, la coherencia de la verdad implica su necesaria consistencia con todo lo que es tenido por verdadero, es decir, la verdad es en el fondo una y universal. Ahora bien, si la verdad como coherencia conduce a la coherencia misma de la realidad, la universalidad de lo verdadero tiene también unas consecuencias onlológicas (cfr. ST 63). La unidad·de la verdad es inseparable de la coherencia y unidad de todo lo real, a pesar de su aparente multiplicidad. Si la teología ha de demostrar la verdad de la doctrina cristiana, esto consiste ante todo en mostrar su potencialidad para explicar satisfactoriamente el mundo y la hisloria universal en su totalidad (cfr. ibid.).

Esto nos conduce a un tercer aspecto de la verdad: la verdad en cuanto universal tiene, según Pannenberg, un carácter fundamentalmente histórico. Una verdad universal tiene que dar cuenta no solamente del mundo natural, sino también de la historia. Pero al mismo tiempo, toda pregunta y toda respuesta humana por la verdad es algo que acontece en la historia y no fuera de ella. Frente a la concepción helénica de la verdad como identidad intemporal de la cosa consigo misma, Israel ha entendido la verdad como constitulivamente histórica (WW 203ss). Esta concepción judía de la verdad ha ganado actualidad en la modernidad, pues tras el descubrimiento de la relatividad de las culturas no ha quedado orra alternativa que admitir el carácter histórico de la verdad y de su unidad (WW 60-73). Por eso, la pregunta teológica por la verdad de la religión cristiana ha de plantearse justamente en el horizonte de la historia (cfr. ST 69).

El problema entonces es aclarar cómo la verdad puede ser al mismo tiempo histórica y universal. Para hacer esto hemos de preguntamos primero qué entiende Pannenberg por historia.

\section{El concepto de historia}

a) Historia sagrada e historia profana. A la hora de abordar el tema de la historia, Pannenberg se halla situado, como la teología europea en general, ante 
el problema de su secularización por la filosolía ilustrada de la hisloria (KG 79ss; TR 658ss) ${ }^{3}$. Por una parte, la historia humana se entiende como una realidad propia, independiente de la historia sacra de los relatos bíblicos. Por otra parte, los mismos texios biblicos son sometidos a los modernos métodos histórco-críticos y hermenéuticos que, aunque de suyo nacidos a partir de la renovación protestante de la comprensión de la Escritura (cfr. HTH 127s), terminan por reducir la historia de la revelación cristiana a un capílulo más de la historia profana, sometida a sus reglas y carente por tanto de toda pretendida uranscendencia.

Ante este hecho, la teología puede tratar de diversos modos de mantener la posibilidad y el sentido de la revelación. Una posibilidad es la subjetivización de la historia de la revelación mediante la distinción entre suceso e interpretación. Este sería el intento de Cullmann: mientras que a los acontecimientos históricos tenemos un acceso positivo por medio de la historiografía, las interpretaciones pertenecen al ámbito de la subjetividad y, en el caso del cristianismo, al ámbito de la fe. La fe selecciona determinados sucesos y les da una determinada interpretación obteniendo así, en un nivel distinto e independiente de la historia profana, una verdadera historia sagrada. La consecuencia incvitable es la necesidad de admitir fuentes para la fe fuera de la historia prolana y el consiguiente dualismo de dos historiast.

Otra posibilidad es la negación misma de la relevancia de la historia para el mensaje cristiano. Esto es, a los ojos de Pannenberg, lo que sucede con las teologías dialécticas o existenciales. La revelación aparece en ellas como una superación (Aufhebung) de la historia y del tiempo cn nombre de la eternidad (Barth) y solamente la historicidad como existencial del hombre individual cobra un significado como escatología actualizada (Bultmann). En cualquier caso, el desalío de la moderna conciencia histórica no es consccuentemente enfrentado ni integrado en la teología, sino más bien rechayado, con lo que la dualidad entre historia profana y revelación pernanece intactas.

Una tercera posibilidad, que Pannenberg denomina "perspcctiva históricosalvílica", entiende la historia entera de la humanidad como un desarrollo de la revelación de Dios a través del progreso religioso de las distintas culturas, evitando de este modo la dualidad entre dos historias. Historia profana e historia de la revelación serian, por tanto, coextensivas, como Rahner ha propuesto ${ }^{6}$. El problema de esta perspecliva es cómo explicar el carácler especial y definitivo de la revelación judeo-crisliana evitando su equiparación con las demás religiones. Según Pannenberg, no basta con proclamar una parte de la historia universal (la historia de Israel y del cristianismo) como "revelación oficial", desde la cual se puede enjuiciar el valor de las demas cosmovisiones, pues esta proclamación es inevitablemente parcial y dogmática?.

Esta última posibilidad es, sin embargo, la más cercana al planıeamiento de 
Pannenberg: para éste se tralará de mantener la perspectiva histórico-salvifica, pero sin caer en la afirmación dogmática de una parte determinada de la historia como revelación oficial: la revelación no es para Pannenberg un hecho sobrenatural, solamente accesible mediante el don de la fe otorgado a unos individuos determinados. Si la teología ha de mantener la verdad del mensaje cristiano, la revelación ha de ser un hecho universal y accesible a todos ( $c f r$. OG 98ss). Esto no quiere decir en absoluto que la revelación sea un producto de la razon humana (cfr. OG 100), pero si es la revelación histórica de Dios la que funda la fe no al revés- - la verdad revelada del cristianismo ha de poder mantenerse como tal en una discusión racional con otras posibles cosmovisiones para así poder presentarse como digna de le.

Ahora bien, para entender qué significa esto más concretamente hay que preguntarse cómo entiende Pannenberg la estructura y el dinamismo de la asi llamada historia profana, para ver en qué modo esa historia puede ser ámbito de la revelación de Dios.

b) La historio como tradición. La tesis de la unidad de la verdad y de su historicidad ha de conducir en principio a la alimación de la unidad rea! de la historia. Ahora bien, esta unidad no es, según Pannenberg, la unidad de un sujeto realizador de la historia, tal como ha sido el planteamiento típico de la Ilustración (cfr, MG 488s, TR 667, EGS 479-481). A la larga, esta idea se acaba disolviendo en la pluralidad de los pueblos y de los individuos, como bien muestra el nistoricismo (cfr. HG 37), pues de hecho, para Pannenberg, solamente los individuos pueden ser considerados como sujetos (cfr. ST 117; WH 352; HG 73). El concepto ilustrado de "género humano" no es en realidad un concepto histórico, sino biológico (cfr. EGS 489; WH 352), que no dispone de las propiedades que permiten hablar de un "sujeto" en sentido estriclo (cfr. WH 353).

Y es que, más radicalmente, antes de la historia ( $c f r$. MG 474s) no hay ni siquiera sujetos individuales, sino que el hombre mismo se constituye como tal justamente a lo largo de la historia (cfr. MG 488). La "histricidad" misma de la que nos hablan Heidegger o Bultuman no es un existencial insito al hombre desde el principio de los tiempos, sino una dimensión del mismo aparecida en su historia (cfr. MG 478-481). Por wdo ello, los sucesos hisióricos no son simplemente productos de un hacer humano consciente y subjetivo, sino que consisten más bien en "acontecimientos" (Widerfahrnisse) que al hombre le sobrevienen sin que pueda disponer sobre ellos ( $c f r$. MG 490, TR 667s). En consecuencia, todo intento de planificar la historia, como por ejemplo en el socialismo, esta condenado al fracaso (cfr. MG 491).

Pero, si no es recurriendo a un sujeto, ¿cómo se puede mantener la unidad de la hiswria? La respuesta de Pannenberg es que la unidad de una determinada siluación histórica viene dada, no por la unidad de quien ha realizado las acciones en cuestión, sino por la unidad coherencial del recuerdo (cfr. MG 490, 
492s). Solamente cuando los acontecimientos históricos son reasumidos en una narración cobran éstos su verdadera unidad. Así por ejemplo, la unidad de la historia de Israel no se explica mediante la continuidad material de un determinado sujeto humano a través de todos los vaivenes históricos (exilio, etc.), sino más bien por la recuperación reflexiva de esa historia de las diversas historias bíblicas. El hombre no es el sujeto de la historia, sino aquél sobre quien ésta se narra (cfr. MG 493ss).

La historia no es, por tanto, el conjunto de actividades o acciones del género humano, sino más bien un proceso de formación de identidad: las narraciones cuentan justamente la configuración de la identidad de personas y pueblos, quienes a su vez reciben y renuevan su identidad a través de estas narraciones ( $c f r$. MG 494-501; TR 668), tal como Lübbe y Habermas han puesto de relieve ${ }^{\theta}$. Por esto dirá Pannenberg que el hombre no es el sujeto, sino el tema de la historia (cfr. EGS 481s; WH353s, MG 497). En esta perspecliva no cabe duda de que la historia, en cuanto proveedora de identidad y de totalidad para los hombres, afecta a su felicidad y salvación (Heil) en el sentido más amplio del término.

En el fondo de estas consideraciones de Pannenberg está la idea de que las vivencias de sentido tienen prioridad sobre las acciones (cfr. MG 499): los sucesos históricos por si mismos son "hechos brutos" aislados y sin continuidad. La conexión y el sentido de los mismos solamente aparece cuando a estos hechos se les añaden sus respectivas interpretaciones (cfr. HG 67, 63; KG 82s; HTH 134-36). Ahora bien, Irente a Kahler que puso de relieve la unidad indisoluble entre hecho e interpretación, Pannenberg rechaza la posiblidad de fijar una determinada interpretación, declarándola insuperable (cfr. TR 663; HG 61s; KG 79). Las interpretaciones se suceden unas a otras en el flujo de la tradición, pero frente a los hechos históricos positivos (por otra parte nunca alcanzables, pues toda investigacion histórica está inevitablemente ligada a determinadas interpretaciones), éstas tienen la ventaja de su continuidad.

Por todo ello dirá Pannenberg que "el proceso histórico es esencialmente un proceso de tradición (Ūberlieferung ). Todos los sucesos políticos e incluso los mismos hechos naturales que conciernen a la historia alcanzan solamente sentido y significado por referencias a las tradiciones en las que vive la sociedad por ellos afectada, y esto con frecuencia en cuanto que las formas de tradición con las que se encuentran son puestas en tela de juicio, dando lugar a su cambio y reconstruccion" (KG 86; cfr. KG 88; ST 254 nota).

c) Hermenéutica de la historia y teología. Esta visión de la historia nos sirve para comprender más concretamente el papel de la teologia Si la verdad que esta disciplina busca tiene un carácter histórico, vemos ahora que esta verdad no puede hallarse en otro lugar que no sea el mismo proceso de transmisión y tradición de distintas interpretaciones del mundo. La verdadera interpretación de la historia solamente se puede encontrar en la historia misma ( $c f$. TR 668). Si la 
doctrina cristiana ha de demosuar su verdad, no pucde hacer esto más que desde la historia (cfr. HG 55, 59). Esto significa que la teología tiene de suyo un carácter fundamentalmente hermeneútico .

No se trata, sin embargo, de una hermenéutica meramente psicológica o existencial, en el sentido de Schleicrmacher, Dilthcy o Bultmann (cfr. HU 94105), sino más bien de una continua interpretación en el scntido de lo que Gadamer denomina "la mezcla de horizontes" (Ilorizont-verschmelzung; cfr. HU 106-116). La historia misma, en cuanto la historia de la tradición (y no una mera simpatía psicológica o existencial con situaciones o autores precedentes), es lo que une el pasado con cl presente (HU 104, 111; HTH 128s) en el proceso permanente de recepción y construcción de lo transmitido por la uradición (HTH 152). Ahora bien, Pannenberg considera que Gadamer, al soslener que el horizonte lingüístico incluye tambićn lo no dicho, termina por desvalorar la estructura predicativa de! lenguaje (cfr. HU 112ss, 118), lo que se cvila si el nuevo horizonte, que resulta de la reinterpretación del horizonte pasado desde cl horizonte presente, es formulado explícitamente (cfr. HU 109s). Esto conduce por extensión a la necesidad de construir bosquejos de la historia universal ${ }^{9}$ como horizonte global de toda interpretación, pues solamente así es pensable la continuidad con el pasado y con el posible significado actual de un texto antiguo (cfr. HU 119-20), en nuestro caso, el posible significado y verdad de los textos biblicos.

Por ello, el teólogo o el creyente en general no disfrutan de especiales privilegios respecto a los hiscoriadores, pues tanto unos como ouros han de buscar la verdad "desde abajo", es decir, desde la investigación científico-hermenéutica de la historia (cfr. HG 54ss, HTH 132ss). La fe no es punto de partida que ya asegura de antemano la verdad de lo que se investiga. Al contrario: solamente el conocimiento de las promesas reveladas hace posible la le en las mismas (cfr. OG 102; HG 66). No se trata, claro está, de una comprensión positiva de este cumplimiento, en el sentido de exigir, por cjemplo, la realización exacta y comprobable de lodos y cada uno de los anuncios bíblicos (cfr. EGS 487s). Si la historia es un proceso permanente de formación y revisión de las tradiciones, tal como hemos visto, el verdadero significado de un acontecimiento (y, por tanto, también de la historia en su conjunto) está siempre abierto a nuevas interpretaciones y reinterpretaciones dentro de la corriente de la tradición (cfr. EGS 488 90).

En realidad, para Pannenberg no hay una diferencia esencial entre la hermenéutica histórica o teológica (cfr. HTH 133ss, 140). Si el carácter histórico de la verdad remile al teólogo al estudio científico de la historia, el carácter universal de la verdad significa que, de un modo u otro, lodo historiador y lodo cientúfico han de preguntarse por la verdad de la realidad y de la historia en su conjunto, es decir, están remitidos de algún modo a las preguntas de la teología. 
El historiador por ejemplo, no puede entender un acontccimiento aislado más que en un marco general, y este marco general a su vez solamente tiene su sentido último dentro de una perspectiva universal que explique la historia (y la realidad) en su conjunto (cfr. MG 500s; HTH 140; HG 69ss; KG 89 nota; TR 669). La dificulad que entraña la elaboración de esbozos de la historia universal consiste en que, en realidad, la historia entera solamente puede contemplarse desde su final, como ya sefhalaba Dilthey (cfr. HTH 143s). Si se quiere evitar el relativismo que resultaría de este hecho, toda doctrina que pretenda verdad (y por tanto universalidad) ha de remitirse a algún tipo de anticipación de la unidad de la historia en el futuro (cfr. HTH 144; MG 496, 500, 510, EGS 490). La anticipación es por eso lo que permite la síntesis entre la universalidad y la historicidad de la verdad.

Con esto hemos visto en qué sentido y bajo qué condiciones una determinada cosmovisión puede pretender ser verdadera. Ahora es menester preguntamos si la revelación judeo-cristiana cumple estos requisilos y si lo hace mejor que otras pretendidas explicaciones de la totalidad del mundo y de la historia.

\section{La verdad del cristianismo}

a) Las religiones y la filosofía. Desde la perspectiva de Pannenberg, toda cosmovisión que aspire a la verdad, como sabemos, tiene que presentarse como universal. El relativismo no es para él una mera renuncia a la universalidad: en la medida en que se desentiende de la universalidad renuncia también a su coherencia y, por lo tanio, a la verdad en sí misma. El desarrollo de la filosofía moderna con su afirmación de la espontaneidad de la razón humana ha conducido en opinión de Pannenberg a que la unidad de la verdad y la correspondencia entre sujeto y mundo solamente pueda ser garantizada (cartesianamente) mediante el recurso a Dios (WW 210-216). La idea de Dios es justamente lo que permite pensar la unidad de la realidad ( $f$ r, ST 81s): "solamente Dios puede ser el lugar ontológico de la unidad en el sentido de coherencia como unidad de Iodo lo verdadero" (ST 63). En este sentido cabe decir con Agusún que Dios es la verdad misma ( $c f r$. ibid). Esto sucede también en el caso de la historia. Una vez que se ha superado lo que Pannenberg considera el mito ilustrado del "sujeto de la historia", solamente la idea de Dios como lugar de la totalidad de la realidad puede fundar su unidad (cfr. HG 73ss; KG 89 nota; HU 92s, 102; HTH 137s), la cual es, por otra parte, presupuesto de loda verdad.

Ahora bien, ¿de dónde se puede obtener esta idea de Dios? Pannenberg hará aquí una importante distinción: para obtener una determinada concepción de lo que sea Dios no se puede recurrir sin más a la experiencia religiosa. Toda experiencia religiosa presupone ya más bien una idea de Dios, que funciona justamente como insurumento interpretativo de la misma (cfr. ST 76ss). La experiencia religiosa le va a servir a Pannenberg para enfrentarse con la cuestión de 
la realidad que corresponde a la idea de Dios, pero el contenido de la misma se ha de obtener más bien de la así llamada "teología natural" (cfr. ST 80). Esta no puede demostrar la existencia de Dios a través de sus pruebas, tal como fue su pretensión clásica ( $c f r$. ST 101ss), pero sí puede mostrar dos cosas sumamente importantes.

En primer lugar, la historia de la "teología natural" muestra que las llamadas pruebas de la existencia de Dios estaban en realidad vinculadas a una determinada concepción de la divinidad vehiculada por las religiones tradicionales. En segundo lugar, las "pruebas antropológicas" han puesto de relieve el carácter esencialmente religioso del hombre, de modo que algún tipo de conocimiento (no necesariamente explícito o temático) de Dios es un elemento constitutivo del ser humano (cfr. ST 104s). Esto es, por lo demás, una tesis de la revelación cristiana misma: Dios es conocido por los hombres a través de las obras de la creación (Rom 1, 19s; cfr. ST 121). Este conocimiento o notitia innala de Dios es una mera constante antropológica: en todo ser humano, antes de existir una distinción temática entre objeto y sujeto, se da en el ámbito del sentimiento una referencia no temática a la totalidad de la realidad (cfr. St 125) como "horizonte abierto del infinito" (cfr. ST 127). Con el desarrollo de la experiencia individual $y$, sobre todo, a lo largo de la historia de las religiones, ese primer conocimiento no temático va tomando una forma y un perfil concreto (cfr. ST 128ss).

Esto significa que tanto una filosofía consciente de su propia historicidad y de los límites de las "pruebas" clásicas ( $c f r$. HTH 152), como también una teología que lome en serio la cuestión de la verdad de la religión cristiana están remitidas a comprobar la verdad de la idea de Dios que nos proporcionan las religiones, pues es en ellas donde toma forma historica concreta la respuesta a la cuestión de la divinidad como fundamento de la unidad de lo real (cfr. HG 75; TR 668). Lo que sí puede hacer la llamada "teología natural" es proporcionar un "concepto marco" sobre los elementos que ha de tener una respuesta válida a esta cuestión en forma de criterios (cfr. ST 120): así, por ejemplo, la divinidad como fundamento de la unidad del mundo ha de ser por luerza única y libre de contradicciones, con lo que el politeísmo queda en principio excluido.

b) La historia como revelación de Dios. Esta perspectiva lleva naturalmente a considerar las religiones bajo un punto de vista muy concreto: no en cuanto cultus deorum (Cicerón), sino, siguiendo a Agustín, como conocimiento de Dios (cfr. ST 134s). Tanto es asi, que para Pannenberg lo que funda todas las demás formas de comportamiento religioso y de culto es ese conocimiento (cfr. ST 189). La religión es, por tanto, más allá de sus diferentes concreciones, el lugar de la pregunta por lo infinito de lo real en cuanto ámbito donde todas la cosas funitas se "recortan" y cobran su realidad ( $c f r$. ST 154ss). Las distintas religiones particulares han de ser sometidas a la pregunta de si sus representaciones concretas de la divinidad son capaces de manifestar en la realidad lunita ese hori- 
zonte infinito que las constituye. Los dioses de las religiones se han de mostrar en la experiencia histórica de la humanidad como los poderes que pretenden ser (cfr. ST 183s). Esta auto-afirmación (o lracaso) de los dioses en la historia de las religiones no es para Pannenberg otra cosa que la historia de la manifeslación misma de la divinidad (cfr. ST 186), es decir, la historia de la revelación.

Semejante concepción de la revelación de Dios no es, para Pannenberg, contradictoria con la concepción blblica de la misma. Ciertamente, Dios no puede ser conocido por los hombres si él mismo no se da a conocer. Pero con esta tesis cristiana fundanental no está dicho por medio de qué tipo de revelación Dios se manifiesta a si mismo. Por una parte, hay que observar que la misma revelación biblica da por supuesta una cierta idea de lo divino, común con otras religiones, como punto de partida para hablar del Dios de Israel (cfr. ST 207ss). Por otra parte, aunque el pensamiento biblico mancja ideas de lo que sea la revelación, éstas acaban conduciendo, en la historia de Isracl, también a la convicción de que es la experiencio histórica de la humanidad el ámbito donde la verdad de la divinidad de los dioses se ha de verificar (cfr. ST 215-234).

La misma historia de la teología conduce a este enfoque de la revelación: una vez que la autoridad de la Escritura, que unía la ideal de la revelación con la de la inspiración divina, no es sin más evidente, y una vez que las vías filosóficas para el conocimiento de Dios han hecho crisis, la tcología ha de pensar la autorrevelación de Dios en el conjunto de la experiencia historica de la humanidad. Esto no significa necesariamente una identilicación con la filosolía hegeliana, pues ésta desconoce su propia linitud y por tanto la necesidad de una anticipación del final de la historia como condición para pensar la verdad en su totalidad (cfr. ST 245-250). La apelación por parte de algunas teologías a la "palabra de Dios" ha de entenderse dentro del marco más general de la historia como revelación de Dios, dado que la palabra de Dios, tal como aparece en la Escritura, no se refiere tanto a Dios mismo como a determinados acontecimientos históricos ${ }^{10}$, los cuales, por otra parte, deciden sobre el carácter de "revelación de Dios" de un determinado discurso profético. La idea de una revelación indirecta de Dios mismo a través de la historia tiene la ventaja de poder integrar las distintas concepciones de la revelación que se hallan en la Escritura dentro de una perspectiva más amplia, abarcadora tanto de palabras como de hechos salvíficos (cfr. ST 251-275). Las palabras aisladas solamente encuentran su sentido en un conjunto más amplio de significado, el cual últimamente remite a la historia en su totalidad. La concepción de la historia como plan y revelación de Dios siúa a las palabras y evila una comprensión aislada y, en definitiva, mítica de su significado (cfr. ST 275-281).

c) La verdad de la religión cristiana. Una vez llegados a este punto podemos abordar ya directamente lo que Pannenberg ha de inido como la cuestión central de toda teología: el problema de la verdad de la religión cristiana. Tras el cami- 
no recorrido, puede decirse que la pregunta que nos hacemos se plantca concretamente del siguiente modo: ¿en qué sentido pucde decirse que la religión judeo-cristiana, en el conjunto de la historia de las religiones, entendida como historia de la búsqueda y revelación del rosuro verdadero de la divinidad, representa la forma más avanzada y plena de la auténtica revelación de Dios?

La respuesta a esta cuestión no puede lograrse según Pannenberg mediante la afirmación dogmática de una determinada rcligión, que se consideraría a sí misma como la "revclación oficial", sino solamente mediante la comparación racional y crítica entre las diferentes religiones y su capacidad de explicar la realidad en su totalidad. Evidentemente, toda posible comparación de la religión cristiana necesita de un desarrollo sistemático de sus tcmas propios, desarrollo en el cual ya se pone a prueba la consistencia de esta cosmovisión y su capacidad de dar cuenta del muncto y de la historia. Ya que aquí no podemos dar cuenta de todos los desartollos sistemáticos de Pannenberg, nos limituremos a poner de relieve lo que según ćl distingue a la religión cristiana y la capacila para cxplicar cabalmente la wolalidad de la realidad en comparación con las ouras religiones.

En las religiones primilivas cncucnura cl hombre el centro de su vida y los criterios para su organización en el orden social, que aparece como fundado por la divinidad. Solamente en las culturas ınás avanzadus se comienza a pensar a la divinidad no solamente como garante de este orden, sino también como principio de sus cambios (cfr. MG 477). Pcro cstos cambios se entienden en un principio como mera reconsurucción dul orden originario. La misma idea de elección divina nunca es aplicada en las culturas extrabiblicas a un pucblo entero, sino solamente a su cabeza; la elección del rey sucede dentro del marco del orden establecido por los dioses con la crcación y este orden como tal no es puesto en tela de juicio (cfr. MG 479s). En cste sentido, ninguna religión habría alcanzado la perspectiva histórica característica de Isracl. Los mismos griegos, por mucho que hayan ya esbozado algunos elementos fundamentales del método historiográfico (pensemos cn Hcrodoto), nunca habrían pensado al hombre mismo y su relación con la divinidad como algo histórico (cfr. EGS 486); así, por ejemplo, para Aristócles los cambios históricos no afectan a la sustancia del hombre ( $c f r . M G$ 484).

Lo característico de Isracl frente a las otras religiones scría precisamente su descubrimiento de la historia como marco formal de la revclación. En este pun10, Pannenberg sigue muy de cerca a $G$. von Rad, pues cntiende que éstc, al abordar el desarrollo histórico de la tcología vetcrotestamentaria, habría superado el dualismo clásico entre kerigma e historiogralía, característico del enfrentimiento modemo con los textos bíblicos: scgún von Rad la historia de Israel no scría en realidad otra cosa que cl desarrollo histórico de sus tradiciones en un proceso continuo de recepción y de renovación, coincidiendo así con la concepción de la historia que tienc Pannenberg, scgún vimos (cfr. KG 84-89). 
Pues bien, desde las primeras narracioncs "yahvistas" hasta los profetas postexílicos, Isracl ha intcrpretado su elccción por Dios de acucrdo al esquema de "promesa y cumplimiento" (cfr. HG 24ss; EGS 488). La continua necesidad de interpretar este esquema a través de las diversas vicisitudes históricas habria conducido a la apocalíptica a situar el definitivo cumplimiento de las promesas al final de la historia. Esto proporciona a la religión histórica de lsrael también una perspectiva universal: Como ya muestran las cronologías del libro de las Crónicas, la historia comienza con Adán (cfr. HG 26), con lo que la creación entera es pensada como historia (cfr. HG 27ss). Esto es justamenle lo propio de la religión judia: la totalidad del mundo que la divinidad funda es una totalidad hisĹrica.

Sin embargo, el universalismo de la apocalíptica no logra supcrar el particularismo de una elección parcial ( $c f r$. EGS 482). Esto sucede solamente en el Nuevo Tesiamento. En él persistc, claro estí, la continuidad con las promesas veterotestamentarias, pero éstas ya no apareccn ligadas a la Torá, sino a la persona de Jesús (cfr. HG 29-31). El fracaso de éste y su mucrie en la cruz no significan para Pannenberg el final del csquema promesa- cumplimiento y con ello la superación de la historia, como sostendría, por cjemplo, Bulumann, sino más bien la forma inesperada de cumplimicnto; cs decir, una especie de corrección al esquema, cosa no solamente caractcrística del Nucvo Testamento (cfr. HG 35). Esta forma inesperada de cumplimicnto es jusumente la resurrección de Jcsús, punto en el que se concentra para Pannenberg la capacidad explicativa de la religión cristiana y su superioridad respecto a otras cosmovisiones y a otras concepciones de la divinidad.

Pannenberg pretende llevar a cabo una Icctura del Nucvo Testamento que haga justicia a uno de los descubrimientos excgéticos más importantes y, a la vez, teológicamente más problemálicos sobre el mensaje de Jesús: su carácter escatológico. Jesús habría anunciado la inmediate: de la venida del reino de Dios como final de los tiempos, ligando a la vez íntimamente esta venida a la aceptación de su persona. Según Pannenberg, para entender esto es necesario partir de la hipótesis de que la resurrección de Jesús fue un hecho histórico, si es que realmente se pretende que tenga significado para nosotros (cfr. WW 221). Orra cosa es saber qué sucedió en realidad con la resurrección: debido justamente a su carácter escatológico no podernos decir en qué consistió exactamente este hecho (cfr. HTH 156). En cualquier caso, la relevancia de la resurección está precisamente en su carácter proléptico: la resurrección de Jesús no es otra cosa que la aparición del final de la historia en el interior de la historia misma (cfr. OG 103ss; HG 42; HU 153-158; WW 220s; ST 228s). En la resurtección de Jesús como anticipación del final de los tiempos residiria justamente lo central del mensaje cristiano y su capacidad para mostrar su verdad, dando cuenta de la totalidad en comparación con ouras religiones y cosmovisiones. 
La mayor capacidad explicativa de la religión judeo-cristiana queda, por una parte, ya de manificsto en su conciencia de la historia. Evidentemente, esta conciencia no es solamente lípica del Antiguo Testamento, como a veces se pretende, sino también del Nuevo. Ahora bien, lo propio de la teologia neotestamentaria sería no solamente la conciencia histórica, sino la comprensión de la historia desde el final (cfr. HTH 144-51). Como vimos a propósito de Dilthey, la anticipación del final de la historia era una condición ineludible para poder pensar la universalidad de una verdad entendida como histórica. La resurección le posiblitó a Pablo entender a Jesús cómo el "nuevo Adán", esto es, interpretar el conjunto de la historia no desde la nawraleza o desde unos origenes míticos, como es todavía el caso de las narraciones del génesis, sino desde su final (cfr. MG 482ss). Si bien el naturalismo helénico echó a perder esta conciencia a lo largo de la historia de la teología cristiana, la crisis modema del concepto de la naturaleza y de los mitos dcl estadio originario (que aún se encuentran en el marxismo) hacen posible y necesario volver a entender la realidad como historia y la historia desde su futuro (cfr. MG 485ss).

La ventaja de esta perspectiva consiste, por una parte, en que, frente a su disolución moderna en el relativismo y en el historicismo, la unidad de la historia queda aquí salvaguardada (cfr. HG 36-44). Desde Jesús se puede entender adecuadamente la cotalidad de la historia (cfr. HTH 139), anticipando su final pleno, y no solamente el conjunto de la propia vida mediante la anticipación de la muerte, al esulo de Heidegger (cfr. HTH 144-151). Por otra parte, frente a las concepciones deterministas de la historia, el futuro queda en ciento modo abierto. Las filosofías deterministas entienden la historia como desarrollo de algo ya prefijado desde el comienzo de la misma (por ejemplo en la naturaleza humana, o en la lógica del espíritu absoluto), con lo que el futuro queda en cierto modo abolido por estar ya dado virtualmente al comienzo de los tiempos. La consideración de la historia desde un futuro anticipado en la resurrección deja abierta la forma concreta de ese futuro, pues la anticipación, en cuanto tal, ni agosa ni determina el contenido de lo que ha de venir (cfr. HG 42-44, 48; HU 120ss; WW 219s; HTH 154ss).

De este modo, la resurrección de Jesús, en la medida en que proporciona una concepción unitaria, universal y abierla de la historia, hace posible la unidad total de la verdad, tal como ésta fue escriba. Justamente por ello se puede concluir la verdad de la religión cristiana ( $c f r$. WW 222) y su superioridad respecto a ouras concepciones de la btalidad desprovistas del mismo potencial integrador ( $c f r$. HG 76-78). En la medida en que esa revelación de la divinidad es solamente una anticipación, puede decirse, sin embargo, que la verdad de la religión cristiana está aún en juego hasta su corroboración plena al final de los tiempos (cfr. ST 281).

Si la doctrina cristiana es la verdadera, ella es ambién la más apta para 
proporcionarle al hombre la totalidad y la identidad en que la salvación (como vimos) consiste. La historia de la revelación como historia de las religiones es entonces también via animae liberandae (cfr. EGS 488). Por eso dirá Pannenberg que la historia de la salvación no es otra cosa que la historia de la tradición (cfr. KG 88) en el sentido arriba expuesto. La salvación así entendida no es ni puede ser el logro de un sujeto humano creador de la historia, sino solamente don de Dios a través de su revelación (cfr. WH 365s, 357). Esto no significa pasividad del hombre, pues la revelación, en cuanto le indica al hombre cuál es su identidad y sentido definitivo, le proporciona una inspiración para la acción (cfr. HG 74 nota; WH 365). En cuanto inspiración, la resurrección de Jesús es capaz de proporcionar esbozos históricos provisionales de realización individual y social (cfr. HTH 154; WH 355s).

\section{El mundo como historia de Dios}

La visión de la historia que hemos expuesto hace comprensible, en primer lugar, el carácter insuperable de la revelación de Dios manifestada en Cristo resucitado, dado que en él ha aparecido ya el fuluro (cfr. HTH 154-156). Con ello, en segundo lugar, se puede pensar también su divinidad, esto es, su carácter de revelación de Dios. Si Dios es el fundamento de la tocalidad, su manifestación en la historia ha de tener necesariamente el carácter dc anticipación del futuro. Afirmar que Jesucristo es Dios es decir que con él el lundamento único de la realidad se ha revelado definitivamente en la historia humana (cfr. HG 67; HTH 126; OG 107-111; ST 270). Sin embargo, en la medida en que esta revelación consiste solamente en una anticipación, la divinidad de Jesucristo solamente se decidira plenamente al final de la historia (cfr. ST 359).

En realidad, esto mismo puede decirse de la divinidad, no solamente del Hijo, sino de toda la Trinidad. Según Pannenberg, Dios funda la historia desde su venida en el futuro, como permanente motivo para reconstruir la continuidad con el pasado (cfr. HG 74; HTH 139). Por eso es Dios inmanente al proceso histórico de la tradición y a la vez Dios del mismo (cfr. HTH 139). Esta icnsión entre inmanencia y transcendencia de Dios halla su expresión en la doctrina trinitaria de Pannenberg. No podemos entrar aquí en una exposición de detalle. Baste con decir que éste, fiel a su concepción de la historia como revelación de Dios, se niega a deducir las personas trinitarias conceptualmente a partir de una determinada idea de Dios (cfr. ST 283-326), considera que hay que partir de las personas reveladas (urinidad económica) para desde ahí reconstruir la unidad en la trinidad inmanente. Lo que esta reconsurucción muestra es la dependencia de la divinidad del Padre respecto a las otras personas justamente en su monarquía (cfr. ST 326-355)".

Ahora bien, esta divinidad solamente se ha revclado en forma de anticipación, y por ello aún está en juego hasta el final de la historia. El Padre, con la 
creación del mundo y con el envio del Hijo y del Espiritu, ha hecho su propia divinidad dependiente del curso de la historia (cfr. ST 357). Se trata cicrtamente de una decisión libre, pues El no depende del mundo, pero una vez que to ha creado se ha hecho dependicnte del mismo, no solamente en cuanto trinidad económica, sino también como trinidad inmanente misma, cs decir, su divinidad misma y no solo su revelación cslá en jucgo (cfr. ST 358). No se trata, claro está, de que la Trinidad sea algo aún no completo, un resultado de la historia. Pero tampoco se trata solamente de que cl conocimiento de esta divinidad por parte de los hombres esté aún incomplcto y que la verdad plena de la fe cristiana solamente se vaya a comprobar delinitivamenle al final de la historia. Se trata de algo más: según Pannenberg, la misma divinidad de Dios es impensable sin la realización definitiva de su reino al final de los tiempos, esto es, sin la manifeslación definitiva de su poder y de su gloria. Por cso mismo, la historia ha de decidir aún sobre la divinidad de Dios (efr. ST 359).

Semejante afirmación solamente se sostiene si la finitud de lo histórico queda de algún modo no solamentc fundada, sino tambićn integrada en Dios mismo. Esto es posible para Pannenberg justamente en base a su concepción de la divinidad como infinitud. Lo infinito no puede ser simplemente lo opuesto a lo finito, pues de este modo estaría limilado y ya scría linito. Lo infinito ha de integrar dentro de si a lo linito. Esto es lo que Pannenberg denomina, aludiendo a la "infinitud mala" de Hegel, la verdadera infinitud (cfr. 432ss). En este sentido se puede entender mejor la dependencia de la divinidad de Dios respecto a la historia. El Dios trinitario, al crear el mundo, ha integrado dentro de sí el tiempo histórico, ya que su eternidad, al igual que su infinitud, abarca al tiempo mismo (cfr. ST 433ss). Por eso mismo, su dependencia respecto a la historia está "ya" en el final de la historia supcrada, pues su definitiva manifestación tiene, por así decirlo, cfoctos retroactivos, por más que ese final "todavía no" ha sido alcanzado (cfr. ST 472-473).

\section{El futuro del cristianismo}

¿Qué es lo que, a los ojos de Pannenberg, ha de ocurrir en el intervalo que nos separa del final de la historia? Evidentemente, ésu es una cuestión abierta, pues la historia misma no está predeterminada, sino abicrla a la definitiva manifestación de Dios. Sin embargo, sí es posible esbozar algunas líncas generales: Pannenberg nos ha dicho que en la historia tanto la manifestación de la verdad plena del cristianismo como la divinidad misma de Dios esún aún en juego. En la medida que el cristiano dispone de una anticipación en Jesucristo, no "sabe" con certeza lo que en la historia va a pasar, pero sí cree y espera en esa manifestación definitiva de Dios que decidirá definitivamente la lucha entre atcísmo y le (cfr. ST 359).

La historia universal nos aparece por ello como un proceso de unificación 
progresiva de los pucblos, de las culturas y de las religiones (cfr. MG 472; TR 670). En cste proceso pasado, pero también futuro de entrecruzamiento e influencia mutua de las culturas y religiones, se decide, "no sin connlictos y luchas", el verdadcro rosuro de la divinidad ( $c f r$. TR 670). Por eso no tiene sentido cualquier absolutización demoníaca de un determinado pueblo o religión (cfr. MG 515), y de ahí también la importancia que para la religión cristiana tiene la unidad de las distintas confcsiones. En estu continua unificación de las religiones, el cristianismo ha de mosurar sicmpre renovadamente su significado y su verdad (cfr. EGS 489s).

La universalidad propia del cristianismo cs, sin duda, una base [undamental, pues le proporciona una facilidad para calalizar esa progrcsiva unión de religiones y culturas. De ahí el rol permanente y central que en cl futuro han de tener los paises de cultura cristiana, la cultura universalista de la modemidad, y, en concreto, la Europa capaz de unificar la fe cristiana con la herencia cultural de la antiguiedad y, al mismo tiempo, de abrirse a los valores de otras culturas (cfr. TR 670-71). Pcro la historia no está aún concluida. Ella sigue siendo aún, en palabras de Pannenberg, "el campo de lucha entre cl futuro de Dios y las distintas formas del poder del Maligno" (WH 366; cfr. TR 670).

\section{Consideraciones críticas}

Para situar corrcctamente esta valoración crítica es menester comenzar subrayando el alcance que a ésla se le pretende dar. En primer lugar, no pretendo aqui enjuiciar la teología de Pannenberg en el conjunto de todos sus temas, no solamente porque esto no es posible en este espacio. sino también porque, a mi modo de ver, el tratamiento concreto de algunos temas de la tcología resulta en Pannenberg sumamente interesante y fecundo. Tampoco pretendo poner aquí en entredicho su concepción de la historia como horizonte abarcador de toda cuestión teológica, pues me parece que esta intuición fundamental de Pannenberg es correcta. Lo que aquí pretendo valorar es, en principio, solamente el modo concreto en que Pannenberg entiende este horizontc como punto de partida de la teología y las consecuencias enriquccedoras o -más bien-limiladoras que esto tiene para el tratamiento de los distintos temas teológicos ${ }^{12}$.

a) El concepto de reología. A mi modo de ver, en el concepto de teología que maneja Pannenberg se perfilan ya los problemas que afectan al conjunto de su conceptuación de la historia como "horizonte abarcador" de la reflexión teológica. Cientamente, este concepto está fundamentalmente afectado por el problema fundamental al que Pannenberg se enfrenta: el cuestionamiento de la verdad de la doctrina cristiana por el ateísmo modemo. Con esto no se pretende caer en una acusación - bastante superficial por cierto- de que esto es un tema típico de la teología europea, elc. La pregunta por la verdad de la religión cristiana frente al cuestionamiento del atésmo (que oor ciento no es un problema 
exclusivamente europeo) es cicriamente un tema al que toda teología se ha de enfrentar ineludiblemente. Es más, tal como piensa Pannenberg, es importante que la teología no presuponga esta verdad, sino que comience problematizándola y que, por tanto, la cuestión de la verdad del cristianismo haya de ser abordada justamente como punto de partida de la reflexión teológica.

El problema, sin embargo, es, a mi modo de ver, la posible unilateralidad. Unilateralidad en el diagnóstico del problema, pues no cstá dicho que cl ateísmo sea el único ni el principal desafío para el cristianismo. La teología de la liberación ha llamado la atención sobre problemas más urgentes no solamente desde el punto de vista ético, sino también desde un punto de vista estrictamente teórico: la pobreza masiva del llamado tercer mundo como desafío a la credibilidad del evangelio. Pero no solamente el diagnóstico del problema es equivocado: esto sería a mi modo de ver conregible. La unilateralidad afecta a la misma concepción del discurso teórico. El que la teología sea una disciplina teórica (pues solamente así puede evitar tener que tomar prestadas sus ideas fundamentales de ouras disciplinas) no significa ni que la teología sea una disciplina exclusivamente teórica ni tampoco que su dimensión práctica sea una pura consecuencia de sus reflexiones teớricas. Pannenberg no se ha preguniado a fondo por la vinculación esencial que se da entre teoría y praxis ni por las implicaciones epistemológicas que esta vinculación tiene incluso para el tratamiento térico de los temas especulativos. La razón humana liene un momento de opción entre posibilidades $^{13}$, lo cual no solamente determina su constitutiva vinculación a la praxis sino que tiene también consecuencias fundamentales para su relación con la fe: Pannenberg, al prescindir de la vinculación de toda teoría con la praxis, puede prescindir también de las implicaciones epistemológicas para la $\mathrm{fe}^{\mathrm{la}}$, convirtiéndose ésta en un momento derivado de la previa mostración de la verdad.

La hipostasización del discurso teórico respecto a la praxis tiene inevitablemente consecuencias para la comparación con otras religiones. La pretensión de poder mantener la verdad de la revelación cristiana sin necesidad de asegurarla dogmáticamente mediante la afirmación de una "revelación especial" tiene sin duda el valor de enfrentar el problema de la verdad del cristianismo sin recursos fáciles que la salvaguardan de todo cuestionamiento. Pero al mismo tiempo se ignora la dependencia de todo discurso teológico respecto a la praxis histórica de fe en la que está inserto, lo cual en definitiva no es más que una forma de vinculación teoría-práctica a la que no solamente la teología está sometida, sino también las cosmovisiones concurrentes con el cristianismo, por lo que en realidad cualquier discusión o comparación con ellas debería comenzar por hacer consciente este dato epistemológico fundamental que condiciona todo punto de partida.

Si la teorla se desvincula de la praxis, es fácil también comprender su limitación respecto a la correspondencia o adecuación como criterio de verdad. A 
mi modo de ver, si se convierle la correspondencia en un momento derivado de la coherencia, se acaba por sustantivar las consurucciones teóricas y por obtener afirmaciones del tipo "Dios es la verdad", en las cuales la realidad (del mundo) aparece como un derivado de la verdad (Dios). Si, por otra parte, la verdad es algo sometido a un desarrollo histórico, la tesis sobre la dependencia de la divinidad de Dios respecto a la creación cobra un scntido explícilnmente idealista, pues se trata de una dependencia de su divinidad respecto a la revelación histórica de su verdad, como vercmos. En cl caso de los sistcmas formales, tanto el teorema de Gcodel como el de Cohen han mostrado una anterioridad de la realidad matemática posulada respecto a su verdad's La tcología no es un sistema formal, y su misma consistencia, por razón de su objeto, es enormente precaria (cfr. ST 481), por lo quc su verdad (o, si sc quicrc, su credibilidad) exigc algo más que criterios meramente formalcs.

b) El concepro de la historia. Por oura partc, la desvinculación de teoría y praxis no solamente tiene consecuencias cpistcmológicas, sino que también la concepción misma de la historia queda afectuda. La historia es concebida por Pannenberg como historia de la tradición. Cuando Pannenberg afirma la hisloricidad de la verdad no pretende en modo alguno Jecir que esta verdad esté vinculada a la praxis histórica que la sostiene, sino solamente su inserción en el flujo histórico de la tradición: todo discurso humano parte del conjunto de ideas y presupuestos que la tradición le da, ya sea para mantenerlos, para revisarlos, para elaborarlos o para rechazarlos. Fvidentemenic, no es que esto sea falso, pero es enormemente parcial: según la perspectiva de Pannenberg, la consinuidad del proceso histórico viene dada por esta permanente transmisión de la tradición. Con ello, los dinamismos económicos, sociales, políticos, institucionales, etc., no son, claro esiá, eliminados de la historia, pero se convierten en hechos aislados, sin relevancia para la sustancia del devenir histórico.

Dicho en otros términos: a pesar de que Pannenberg ha subrayado la unidad de suceso e interpretación, la continuidad de la historia se da para él solamente en el nivel de las interprelaciones (tradición), no en el nivel de la praxis. Una concepción más sintética de la rclación praxis-teorla hubiera conducido, por el contrario, a una visión más integral de la historia como proceso de posibilitación ${ }^{16}$, lo cual permite integrar en el dinamismo de la historia desde los elementos biológicos y económicos hasla los momentos explícitamente culturales y espirituales. Esto es, dicho sea de paso, fundamental si se quiere pensar la Iglesia como sacramento histórico de salvación y no como mera asociación de transmisores de una determinada tradición.

Pannenberg justifica su concepción de la historia como proceso de tradición en base a la imposibilidad de considerar al género humano como sujeto de la historia. El hombre es tema y producto de la historia, no su sujero. A esto hay que objetar, en primer lugar, que el que el hombre se constituya en la historia no 
niega en absoluto que el hombre sea quien la haga, si se admite el principio ("dialéctico") de interacción como caracierístico de toda praxis. Pero más importante es el hecho de que Pannenberg solamente vea como alternativa a su concepción de la historia como tradición el recurso a un macrosujelo. Ciertamente no hay sujeto humano sin historia y ciertamente el gćnero humano no tiene las características que se predican del hombre cuando se habla de él (justificadamente o no, ése es otro problema) como "sujeto". La cuestión está en que muy bien puede haber una continuidad en la praxis humana a lo largo de la historia sin necesidad de que por eso a los hombres se les convierta en un macrosujeto protagonista y planificador unilario de la historia.

La tesis de la posibilitación sosticne que, por una parte, el hombre es agente (quizás sea este término más adecuado que el de sujcto) de la historia, pero lo es simplemente en cl sentido de que cada individuo, cada grupo y gencración recibe de la anterior un conjunto de posibilidades (no sólo de tradiciones) de acción, que se ha de apropiar, transformándolas en un sentido u otro. Pero lo que constituye la continuidad de la historia no es la permanencia de un sujcto o agente, lo cual no se da y en ello tiene razón Pannenberg, sino la continuidad de la praxis en cuanto posibilitación. Por otra parte, Pannenberg parece pensar que la única alternativa a la idea de un macrosujeto de la acción histórica es la disolución de la misma en un conjunto de operaciones de individuos aislados. A esto hay que objetar que muchas acciones históricas no se entienden sin el recurso a instituciones o grupos supraindividuales. En cualquier caso. no son estos agentes colectivos los que garantizan la continuidad dinámica de la historia, como tampoco lo son exclusivamente sus tradiciones, sino el proceso más amplio de posibilitación. En la historia no hay ni mera continuidad de pensamiento como tradición ni mera continuidad de sujeto, sino continuidad de acción.

De estos presupuesis de Pannenberg se derivan muchas consecuencias que aquif no podemos tratar en detalle. Baste con mencionar el hecho de que su separación entre conocimiento y acción le lleva a pensar las religiones principalmente desde su aspecto cognoscitivo, como búsqueda de la verdad sobre la divinidad, siendo el comportamiento religioso mera consecuencia de ese primer momento cognoscilivo. Si por otra parte la coherencia implica la universalidad de la verdad, no es de extrañar que lo primero que Pannenberg vea en las religiones sea una pregunta por la verdad del mundo en su totalidad. Su reconocimiento del elemento de "confianza" y de fundamentación de la propia vida lo traduce Pannenberg rópidamente en pregunta por la walidad (cfr. ST 126ss). La consecuencia es la necesaria interpretación del politeísmo como monoteísmo primitivo y el subsiguiente desconocimiento del significado fundamental del mismo para la autocomprensión de la fe judeo-cristiana. Si por el contrario se admitiera la unidad profunda entre comportamiento y conocimiento religioso, la cuestión principal de las religiones (y la llamada notitia insita) no sería la unidad de la totalidad de lo real sino la pregunta por el fundamento de la propia 
praxis, con lo que el politeísmo sería una respuesta no solamente posible para quien desconfía de la unidad del mundo, sino también enormemente importante como altemativa al Dios de Istael, y no sólo en el pasado, sino también en la actualidad.

Desde aquí se comprende también su valoración de la experiencia histórica de Dios en las religiones: para Pannenberg la experiencia religiosa individual es interpretada siempre desde la imagen de Dios que una determinada tradición proporciona. Como la praxis humana no es lo que determina el dinamismo de la historia, sino la transmisión de interpretaciones de la totalidad en la tradición, la experiencia histórica de las religiones no es otra cosa que el conjunto de interpretaciones y reinterpretaciones de la realidad sobre el que una religión dispone. Que una experiencia se pueda transmitir a través de la praxis (sin excluir su interprelación, pero en unidad de ambas) pareciera importante para explicar el significado central de la ley en el Antiguo Testamento o del seguimiento en el Nuevo.

c) Carácter derivado de la salvación. Esta concepción de la teología y esta concepción de la historia tienen consecuencias importantes -y aleccionadoras- para su visión de la salvación. La concepción de la historia como historia de la tradición (fruto también del divorcio entre teoria y praxis) conduce inexorablemente a que la historia de la humanidad aparezca para el teólogo en primer término como historia de la revelación y no como historia de la salvación. La salvación es entonces una consecuencia de la revelación de la verdad cristiana, en cuanto ésta le señala al hombre su sentido y le propociona una verdadera identidad. Pero la historia no es en sentido propio historia de la salvación, pues la continuidad dinámica del proceso histórico está constituida por la progresiva revelación de Dios, que consutuye de hecho para Pannenberg la esencia de lo histórico. Por esto mismo puede decirse que, para concebir la "revelación como historia", Pannenberg ha tenido que pagar el precio de entender la historia como revelación, sometiéndola a una mutilación radical que últimamente conduce a la imposiblidad de entender, más que de modo indirecto, la dinámica de la historia humana como una dinámica de salvación.

Este hecho es aleccionador porque muestra cómo en determinadas teologías la espiritualización del sentido último de la fe cristiana (en este caso la espiritualización es una intelectualización) termina por renunciar a elementos fundamentales de la misma, generando un reducionismo en sentido propio de la expresión: la revelación no es una historia de la salvación sino que, por el contrario la salvación es un hecho derivado de la historia, caracterizada por su dinamismo revelador. No podemos entrar aquí al tratamiento individualizado de las implicaciones que esta reducción tiene para cada uno de los temas de la teología. Pero a título de ejemplo basta con señalar que, desde la teologia de Pannenberg, el acontecimiento central de la fe judía, el éxodo, no es relevante por su 
carácter histórico-salvífico, sino solamente por su contenido revelador (cfr. ST 268). Del mismo modo, el sentido fundamental de la misión de Jesús no es la salvación (eso es más bien una consecuencia de la verdad sobre el mundo y la historia que con Jesús aparece), sino el dar a conocer la gloria y el poderío de Dios (cfr. ST 336).

d) El mundo como historia de Dios. Pannenberg ha afimado que la divinidad de Dios está en juego en la historia. Esta tesis necesita de una discusión más detenida.

Uno podría figurarse que lo que está en juego en la historia es simplemente la divinidad quoad nos, nuestro conocimiento de ella, que aún está incompleto y que solamente alcanzará su total plenitud y seguridad al final de los tiempos. Pero Pannenberg sostiene que la dependencia de la divinidad de Dios respecto a la historia va más allá de su mero conocimiento por nuestra parte. Ahora bien. no explica muy claramente en qué consiste ese "más". Según él se trata de que en la historia se "decide" sobre su divinidad. Pero, ¿qué significa este "decidir"? Según Pannenberg, la decisión consistc en la verificación histórica del potencial propio de una determinada divinidad para dar cuenta de los cambios en la experiencia del mundo que sus fieles experimentan (cfr. ST 179). En eso consiste el poder de una divinidad (cfr. ST 174-184), y así se entiende que la revelación del conocimiento del Padre por parte de Jesús consista al mismo tiempo en nıanifesLación de su poder y gloria.

De hecho, entonces, la experiencia histórica de la humanidad, como enriquecimiento constante de la tradición, va comprobando si las distintas imágenes de la totalidad se ajustan a su interpretación del mundo como totalidad. Pero esto no significa más que el decir que el conocimiento de Dios (y si se quiere, nuestra aceptación del mismo) se desarrolla y está en juego en la histonia. ¿Por qué dice Pannenberg que también su divinidad lo está?

Si por divinidad entendemos el carácter de su realidad absolutamente absoluta es dificil pensar que ella se encuentra en juego. Incluso la concepción de Dios como verdaderamente infinito (que a mí me parece mucho más lecunda que la idea de una causa primera) y eterno, capaz por ello de albergar dentro de sí mismo la divinidad y el tiempo (y por lo tanto no meramente contrapuesto contra ellos), implica solamente la tesis de que la realidad de Dins está finitamente afectada por la historia en la medida en que él mismo, en la creación, lo ha decidido así. Pero no implica, sin embargo, que su carácter de Dios (su divinidad) esté plenamente en juego, ya que su realidad infinita, que abarca en sí el mundo y la historia, al mismo tiempo la supera y transciende.

Ahora bien, Pannenberg, consecuente con su perspectiva idealista, ha esbozado otra idea de la divinidad de Dios, según la cual ésta coincidira con la verdad siempre idéntica a sí misma, teniendo esta verdad incluso prioridad sobre 
la realidad (cfr. ST 63). Si, por otra parte, la historia cnicra de la humanidad no es otra cosa que la revelación paulatina ( $y$ anticipadora) de la verdad, es inevitable la consecuencia de que la divinidad misma de Dios (y no solamente nuestro conocimiento de la misma) csté en jucgo en la historia. Divinidad significa en este caso no lanto la realidad de Dios, sino más bien su verdad, aunque queda entonces abierta la pregunta de si la realidad de Dios es anterior a su verdad, lo cual no parece posible dados los presupucstos de los que parle Pannenberg.

Sin embargo, esto no significa propiamente que Dios sea resultado de la historia como culminación de nuestro conocimicuno de la verdad (cfr. ST 359), y por tanto no significa tampoco quc nucstro conocimiento actual se pueda identificar con la verdad misma de Dios (cfr. ST 70-71), como sucedería en la filosofía de Hegcl. La razón es que la verdàd no cstá en "la mente de Dios antes de la crcación del mundo", como diría Hegel, sino en cl Dios que viene desde el final de la historia. La perspectiva escatologica es lo que le evila a Pannenberg la caída en el hegelianismo (cfr. HU 120-22; WW 219s). Esta es la diferencia esencial con Hegel, pero, a mi modo de ver, la unica diferencia esencial. El mismo Pannenberg lo ha dicho con claridad a propósito de la comprensión del libro Offenbarung als Geschichie, "como hcgclianismo tcológico. Puede ser que algunas formulaciones de la introducción hayan favorecido tal malentendido, pero, de hec'lo, de lo que se trataba cra de la solución de las aporías que hay en la formulación de Rothe del concepto de revelación como manifestación e inspiración mediantc la recuperación le la tesis iJcálista de la historia universal como revelación de Dios, pero de modo lal que el concepto idealista de la historia por su parte fue corregido de manera decisiva mediante la idea de una anticipación desde la totalidad de la historia desde su final..." (ST 250).

e) La suerte del cristianismo. Esta perspectiva cscatológica le guarda a Pannenberg de otro peligro cáracterístico del hegelianismo: la identificación de la suerte de la divinidad misma con una decerminada civilización, pucblo o movimiento histórico. Si la verdad viene del futuro, toda la absolutización de un momento presente es una falsificación misma. Pcro de lo que no le libra la perspectiva escatológica es de entender una determinada corricnte de tradición como cl lugar previlegiado de la revelación (si bicn aún no plena) de Dios y por tanto de su presencia. Y cste lugar previlegiado no pucde ser otro que la civilización cristiana, pues en clla la verdad de la divinidad ha presentado su figura más completa y avanzada. De ahí cl importante rol que Pannenberg le ha de adscribir a Europa y a las naciones cristianas en el proceso histórico presente y venidero. Ahora bien, isignifica la fe en la verdad de la religión cristiana la creencia en que la positividad histórica del cristianismo es el lugar previlegiado de la manifestación de Dios? Si la historia es csencialmente revelación, esta conclusión es inevitable, pues Dios estŕ privilcgiadlamente allí donde se revela. $\mathrm{Si}$, por el contrario, la historia de la salvación tiene prioridad y constituye a la historia de la revelación, siendo ésta un inomento de la primera, la religión 
cristiana cn cuanto "conocimiento de Dios" tiene un papel secundario respecto al lugar de la presencia histórica real de esa salvación, que según Mateo 25 son los pobres, independientemente de su adscripción ${ }^{17}$ religiosa o ideologica. Quizás en virtud de esto afirme también el evangelio la prioridad de los pobres no solamente respecto a la presencia real de la salvación sino también en lo que respecta a la revelación misma (Mi 11, 25-27 y par.).

Hace 500 años, las divinidades del contiennte amcricano no pudieron mantener su prectendida explicación de la toblidad, pues, de modo semejante con lo sucedido con el dios sumerio Enil (cfr. ST 178), no accrtaron a explicar los cambios ocurridos en la experiencia del mundo de sus adoradores tras la irrupción triunfante y destructora de los primeros represcntantes de la civilización cristiana europea. La conciencia misioncra y universalisti dcl cristianismo sí fue capaz de explicar e incluso justificar tales acontecimicntos, aunque al mismo tiempo hubo dc condenar todos los excesos no conciliables con los valores mismos de la religión cristiana. El fraile dominico Bartolomé de Las Casas, sin ser teólogo especialmente brillante, entendió a los habitantes de aquel continente como "los Cristos azotados de las Indias". Es decir, pensó que en aquellos infieles se hacía presente cl rostro de la divinidad, y no directamente en la tradición religiosa de quicnes los azotaban. Esta tcsis no se pucde mantener sin alterar radicalmente la concepción de la historia mantenida por Pannenberg.

\section{Notas}

1. Las obras de Pannenberg se citarán según el siguiente esquema de siglas:

EGS: "ErIordert dic Einheit der Geshichte ein Subjekt?", en Geschichie-Ereignis und Erzählung, ed. por R. Koselleck y W.D. Stempel, München, 1973, pp. 478-490.

HG: "Heilsgeschehen und Geschichtc" (1959), cn Grundfragen systematischer Theologie. Gesammelle Aufsätze, L.1, Göuingen, 1967. pp. $22-79$.

HU: "Hermeneutik und Universalgeschichic" (1963), en Grundfragen systematischer Theologie. pp. 91-122.

HTH: "Ober historische und thoologische Hermeneutik" (1967), en Grundfragen systematischer Theologie, pp. 123-158.

KG: "Kurygina und Geschichte" (1961), en Grundfragen systematischer Theologie, pp. 79-90.

MG: Antropologie in theologischer Perspeksive, Göttingen, 1983, 540 pp., especialmente el capítulo "Mensch und Gcschichtc".

OG: "Dogmatische These zur Lehre der Offenbarung". en Offenbarung als Geshcichie, ed. por W. Pannenberg, pp. 91-114.

ST: Systemarische Theologie. 1. 1, Göllingen. 1988, 515 pp.

TR: "Systematisch-theologisch" zur "Geschichte/ Geschichtsschrcibung/ Geschichtsphilosophie". en Theologische Realenzyklopädie, Band XII, Berlin, 
1984, p. 658-674.

WH: "Weltgeschichte und Heilsgeschichte", en Probleme biblischer Theologie, Mürchen, 1971, pp. 349-366.

WT: Wissenschaftstheorie und Theologie, Frankfurt a.M., 1973, 454 pp.

WW: "Was ist Wahrheit?", en Grundfragen systepmatischer Theologie, pp. 202. 222.

2. En el fondo piensa Pannenberg que si la teologla es participación en el saber que Dios tiene de sí mismo, este saber no puede concebirse como práctico, cfr. ST 14.

3. Sobre este problema puede verse mi trabajo "El problema de la historia en la teología de Gustavo Gutiértez", en Revista Ladinoamericana de Teologí, 18 (1989), 335 364.

4. Cfr. WH 358-361, TR 658ss.

5. Sobre K. Barth véase HG 22.60, TR 664; sobre R.Bultunann HG 22, 101-105, TR 664.

6. Cfr. "Weltgeschichte und Heilsgeschichte", en Schriften zur Theologie, t. 5, 1962 , pp.115-135.

7. Cfr. TR 660ss; sobre su crítica a la posición de Rahner cfr. WH 361-363.

8. Cfr. uambiên J. Habermas, "Über das Subjekt der Geschichte. Kurze Bemerkungen zu falsch gestellen Alternativen", en R. Kosellek y W.-D.-Stempel (ed.), Geschichte -Ereignis und Erzählung, München, 1973, pp. 470-476.

9. En un sentido cercano a Hegel, cfr. HU 116-118.

10. Sobre la interpretación de Pannenberg de In 1,1 puede verse, por ejemplo, ST 258.

11. Como muestra el hecho de que el sentido último de la misión de Jesús no es otro que la glorificación del Padre y la manifestación de su divinidad (cfr. ST 336).

12. Por poner un ejemplo: no se pretende aquí enjuiciar su desarrollo concreto del tema de la Trinidad (cfr. ST 283-483), que a mí personalmente me parce muy isspirador. Tampoco se pretende poner en entredicho el hecho de que Pannenberg trate el terna de la trinidad en el marco de la historia, pues éste me parece el marco adecuado y lo que hace su estudio especialmente interesante, sino que lo aquí se busca es solamente preguntarse si su concepción de la historia contribuye a un tratamiento cabal e integral de este tema o si más bien lo limita.

13. Cfr. X. Zubiri, Inseligencia y razón, Madrid, 1983, 354 pp.

14. Cfr. X. Zubiri, El Hombre y Dios, Medrid, 1984, pp. 222 ss.

15. Cfr. X. Zubiri, Inseligencia y logos, Madrid, 1982, pp.133-150.

16. Cfr. X. Zubiri, "Le dimensión históriea del ser humano" Realitas I, Matrid, 1974. pp.11-69.

17. Prescindiendo aquí de la pregunta sobre si ésta es la verdadera esencia de la religión. 